\title{
Scattering microscopy takes single-particle tracking to the next level
}

\author{
Interferometric scattering microscopy enables extended monitoring of gold-nanoparticle-labeled membrane \\ proteins with impressive spatiotemporal resolution.
}

C ellular membranes are rich sites of biological processes that affect cell morphology, movement, signaling, gene expression, and more. Methods that allow the movements of individual membrane proteins to be tracked over time can yield meaningful biological insight and are therefore the subject of intense development. Many such methods are based on fluorescence microscopy, in which a protein of interest is labeled with a dye or fluorophore and tracked over time. While important, these methods are limited by the brightness and photostability of the dyes, which constrain the duration, speed, and resolution of single-particle tracking.

Vahid Sandoghdar, at the Max Planck Institute for the Science of Light, and Richard Taylor, a postdoctoral fellow in Sandoghdar's research group, wanted to bypass the limitations associated with fluorescence microscopy in order to tackle the challenge of tracking membrane proteins with high spatial and temporal resolution over long periods of time in living cells. For this, they and their colleagues turned to interferometric scattering microscopy (iSCAT), a method that Sandoghdar has pioneered for nearly two decades. Sandoghdar notes that the work builds on previous developments from his group that used iSCAT to track viruses and monitor single proteins.

iSCAT is a sensitive technique based on the detection of the light a molecule scatters after illumination, and it has been used to track even single unlabeled proteins. However, tracking becomes quite challenging when it is done in the context of a cell, because the cell gives rise to constantly changing, speckle-like background, making it hard to detect the scattering specific to the particle of interest. To address the challenge of this fluctuating background, the researchers developed and implemented an algorithm that allowed them to extract the full point spread function of the object being tracked directly from each frame. They then determined the movement of the particle by cross-correlating positions between individual frames. Finally, to get the most information possible from the method, the researchers

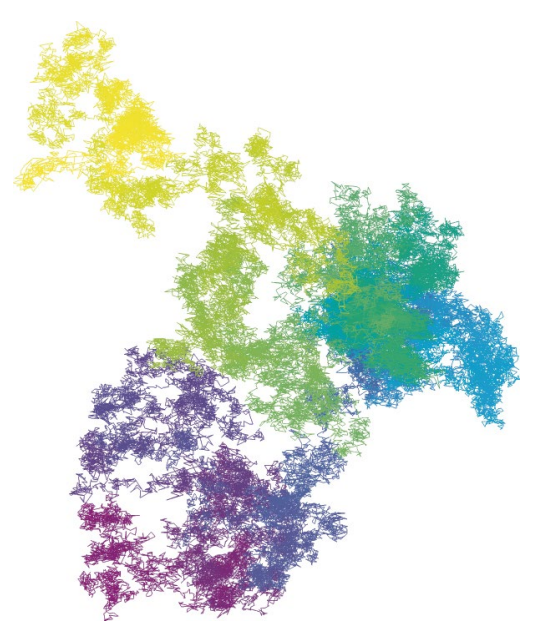

A lateral diffusion trajectory for gold-nanoparticlelabeled EGFR. Reproduced with permission from Taylor et al. (2019), Springer Nature.

pushed the approach into 3D by exploiting distinct ring features of the extracted point spread functions to determine the precise axial position of the tracked particles.

The team demonstrated the power of this approach by tracking gold-nanoparticlelabeled epidermal growth factor receptor (EGFR) in mammalian cells grown in culture, where they were able to monitor nanoscale changes in receptor movements with microsecond time resolution and for more than ten minutes. In these studies, they observed the constrained diffusion of EGFR in nanodomains on the plasma membrane, as well as long-term movement of EGFR along filopodia. In other experiments involving ultra-high temporal resolution, in which 20,000 frames per second were recorded, the researchers were able to observe angular movements of the gold nanoparticle as the labeled EGFR was confined to a clathrincoated pit-a result that Sandoghdar found surprising and hopes to investigate further.

"Following a protein during its diffusion and transport on the plasma and nuclear membranes of a biological cell was always on our to-do list because this is a particularly difficult problem to tackle for conventional fluorescence-based microscopies," recalls
Sandoghdar. However, he notes that there were challenges beyond overcoming the technical issues related to fluctuating background. "Coming from the community of quantum optics and laser physics, it took us a while to master some basic cell biology techniques and to dare applying our know-how to real cells."

Although the method is already broadly applicable, there are developments that could improve the approach. "There are several next steps in many exciting directions, and we certainly will not be able to explore them all. So there is plenty to do for many other groups," says Sandoghdar. He hopes to push the method to monitor even smaller gold particles and to use the method to study viruses, which he notes even unlabeled have strong signal in iSCAT and which are currently under-studied. He also mentions that the method could be combined with confocal fluorescence microscopy. This combination would allow gold-labeled particles tracked with high spatiotemporal resolution to be better placed in the context of fluorescently labeled structures within a cell, for a more complete biological picture.

Their tracking experiments were done on living cells, and when asked about phototoxicity, Sandoghdar explained that iSCAT does not require high light intensities because of the efficiency of light scattering. He did clarify, however, that gold nanoparticles can heat up when illuminated and that higher light doses are needed for faster imaging, so proper controls should be carried out for phototoxicity that are specific to the experimental conditions. Taken together, their results showcase the power of 3D iSCAT for single-particle tracking in cells and should entice biologists to explore these methods and developers to push them even further.

\section{Rita Strack}

Published online: 30 May 2019 https://doi.org/10.1038/s41592-019-0438-3

Research papers

Taylor, R. W. et al. Interferometric scattering microscopy reveals microsecond nanoscopic protein motion on a live cell membrane. Nat. Photon. https:// doi.org/10.1038/s41566-019-0414-6 (2019). 